\title{
Simulation as an Educational Strategy to Increase Medication Error Identification in Licensed Practical Nurses
}

\section{Deryus Tillman DNP, MSN, RN}

Assistant Professor, School of Nursing, Columbus State University, 4225 University Avenue, Columbus, Georgia 31907, United States.

\author{
Article Details \\ Article Type: Research Article \\ Received date: $30^{\text {th }}$ October, 2019 \\ Accepted date: $19^{\text {th }}$ November, 2019 \\ Published date: $21^{\text {st }}$ November, 2019
}

"Corresponding Author: Deryus Tillman, Assistant Professor of Nursing College of Education and Health Professions, School of Nursing, Columbus State University, 4225 University Avenue, Columbus, Georgia 31907, United States. E-mail: dt00111@,georgiasouthern.edu

Citation: Tillman D (2019) Simulation as an Educational Strategy to Increase Medication Error Identification in Licensed Practical Nurses. J Comp Nurs Res Care 4: 151. doi: https://doi.org/10.33790/jenrc1100151.

Copyright: (2019, This is an open-access article distributed under the terms of the Creative Commons Attribution License 4.0, which permits unrestricted use, distribution, and reproduction in any medium, provided the original author and source are credited.

\begin{abstract}
Objective: To introduce a simulated presentation to both validate and enhance competencies in medication error identification and proper reporting.
\end{abstract}

Methods: In this pre-post project design, participants who consented to participate were asked to take a pre-test to evaluate baseline knowledge regarding medication administration and medication error identification. Following the pre-test participants were asked to take part in a one-hour long instructional simulation activity, where they were able to apply their skills of medication administration and while being exposed to possible medication errors. In addition reporting practices were explored. Afterward, participants were asked to take a post-test, which was identical to the pre-test.

Findings: All ten participants were licensed practical nurses (LPNs) with less than five years of experience, who administer medication as part of their daily duties. Nine of the participants $(\mathrm{N}=9)$ were female and one male. $(\mathrm{N}=1)$. Pre-test scores ranged between $78 \%$ and $90 \%$. The analysis revealed a mean score of 84 with a standard deviation of $4.69(\mathrm{SD}=4.69)$ as signified in table 2 . This indicates that on average participants had a score percentage of 84 . Seventy percent of the participants scored at or above $82 \%$. Post-test results following one hour of simulation instruction revealed a percentage score range between $88 \%$ and $97 \%$. The analysis revealed a mean score of $92.4 \%$, with a standard deviation of 2.67 . This indicates that the average post-test percentage score for the sample is 92.4 .

Conclusion: These results imply that simulation instruction does have an effect on medication error identification. Specifically, the results suggest that medication error identification accuracy increases when individuals participate in a one-hour simulation instruction experience. Simulation instruction could be used for ongoing training for practicing nurses. More specifically, the use of simulation could prove to be a plausible resource for the orientation of new nurses with little experience. Appropriate training and practice are essential to confidence, safe practice, and better patient outcomes. The simulation creates a safe environment for these novice nurses to groom skills and learn from mistakes.

\section{Introduction}

The topic of medication errors fuels discussions of both cost and quality in the health care industry as these medication errors have the ability to be catastrophic to patients and the organizations that seek to provide service. From a financial standpoint, these errors cost the United States healthcare system over $\$ 136$ billion annually [1]. While healthcare organizations continue to focus on the reduction of such errors, each year medication errors are thought to account for about 7000 deaths on average in the United States. Reports from The Institute of Medicine [1] concluded that between 2007 and 2011 there were over 30,000 deaths related to medication errors. This issue becomes more alarming as this number increased to more than 45,000 in 2012.

Designing and implementing tailored interventions to reduce medication errors are dependent on the identifying of weaknesses within the system that threaten quality care delivery [2]. To date, research has identified the use of PowerPoints, face to face interviews, surveys, pamphlets, and poster boards as tried modalities for changing the culture of medication error identification and reporting. Yet, research on the use of simulations for the purposes of changing the culture related to medication error reporting has not been fully explored.

\section{Background and Theoretical Framework}

Research suggests medication errors can take place at all points of the medication administration process by violating one of the patients' rights: the right patient, the right drug, the right dose, the right route, and the right time [3]. Being able to identify a medication error accurately is essential to consistent reporting. Continued education to improve knowledge of medication management and administration is an important intervention to reduce medication errors. Nurses must have knowledge and skills in order to recognize potential medication errors before they occur.

A cross-sectional study performed using nursing staff in inner city hospital determined that the lack of a precise definition of medication errors was a chief obstacle to error reporting [4]. This suggests that a nurse's inability to determine what constitutes as an actual error is a possible blockade to medication error reporting.

This explicitly speaks to ethical concepts of beneficence (doing what's in the best interest of others) and non-maleficence (preventing harm). When nurses accurately report a medication error, they are actively engaging in beneficence. Data collected from reporting systems impact nursing practices, by exposing system opportunities for improvements thus preventing future harm.

The theoretical and conceptual framework for this project is three fold. 
Building on Reason's Just Culture Model, Benner's Novice to Expert and the Swiss Cheese Model.

The Just Culture Model focuses on remedying errors and poor patient outcomes not by focusing on individuals but organizational improvement opportunities. This model compliments the ideology behind the Institute of Medicine (IOM) 1999 report "To Err Is Human." The IOM presented the thought of focusing on the health system to make it safer. The report determined that as many as 98,000 patients die each in hospitals as a result of preventable medical errors. The IOM came to the conclusion that errors are not a result of personal carelessness but instead arise from corrupt systems, procedures, and environments [5].

There are two types of errors that impede patient safety. Latent or blunt-end errors arise from design failures or organizational system failures that permit serious errors, while active errors or sharp-end errors are at the individual level and are more readily identifiable [6]. Psychology professor John Reason analyzed these error types and discovered that most accidents are not a result of isolated events, but are the product of basic system flaws [6]. His coined Swiss Cheese Model represents the theory and illustrates how multiple checks can prevent error occurrence. The ideology is that "the slices of cheese are layered, and each layer is a defense against the holes in the cheese, which represent a problem or error in the system. The more layers of cheese, the less likely it is that the holes will line up for an error to occur" [7].

Benner's theory places nursing practice into five stages or levels of clinical competency: novice, advanced beginner, competent, proficient, and expert. These five levels represent an overall change in two aspects of a nurse's skills, increased independence in reliance on abstract ideas and principles and an increase in critical thinking. Experiences gained over time will enable a change in perception.

\section{Research Question}

In licensed practical nurses with less than five years' experience employed in long-term care facilities, does a one-hour staff development experience using simulation instruction, compared to no instruction, improve medication error identification accuracy?

\section{Materials and Methods}

The aim of this study was to answer the question "In licensed practical nurses with less than five years' experience, employed in long-term care facilities, does a one-hour staff development experience using simulation instruction, improve medication error identification accuracy?"

This study followed a pre-post test design. Participants were asked to take a pre-test to evaluate baseline knowledge regarding medication administration and, medication error identification. Following the pre-test participants were asked to participate in a one hour long instructional simulation activity. Participants were then asked to take a post-test, which was identical to the pre-test. Lastly, participants were invited to a debriefing session to discuss opinions and perspectives of the scenario.

\section{Data Collection, Instrument, Testing and Sample}

Inclusion criteria for participation in the project required the LPN to have less than five years' experience, be employed at a long-term acute care hospital (LTACH), and have medication administration duties. There were no age limits for participation. A total of ten participants were recruited.

The Safe Administration of Medication Scale (SAM) Scale was used for both the pre and posttest evaluation. The SAM Scale was developed by Deborah Ryan [8] to measure knowledge and performance objectively during the administration of medication by novice nurses; it is based on the Five Rights of safe medication administration. The SAM includes a total of five cases, each of which has two to three vignettes, and each vignette having five items for a total of 70 items. Kelly Gonzales [9] in a study that compared several evaluation tools for measuring medication administration safety competency, reported that the SAM scale displayed evidence of content validity, fit validity, and reliability of separation with a Cronbach's alpha of 0.77 .

A basic demographic form was used to collect demographic data. The demographic form asked questions regarding; gender, years of experience, medication administration duties, and level of education. This form was completed as part of three pre-testing phase.

The recording of identifying information on the data collection tools was avoided for this project. The demographic form the pretest, and the post-test were numbered. Each participant received data collection tools with a specific number on each set of tools. A sign in sheet was used to determine that each participant received the same number on each tool. The numbers were utilized on each tool as identifiers to maintain confidentiality.

The scores from the pre-test were used as baseline data to determine the current accuracy of the licensed practical nurses. Following simulation instruction on medication error identification simulation, an identical post-test was administered; the scores were collected to determine if the intervention improved medication identification in participants. The variables were identified by codes.

\section{Data Analysis}

The quantitative data was analyzed to determine if the simulation activity increased the accuracy of medication error identification in participants. The potential benefits of this project are to increase the nurse's level of confidence and ability to accurately detect a medication error, which will improve the incidence of medication error. Additionally, it is hoped that this data will lead to discoveries that will improve patient outcomes.

Data was entered into SPSS 24. Descriptive statistics was used to describe the sample and included frequencies, percentages, and standard deviations. For categorical or nominal data, frequencies and percentages were calculated. The information collected on the demographic form was used to complete a demographic analysis. This information was used to confirm that all participants were LPNs who administer medications. Percentage calculations were completed to determine the percentage of male and female participants as well as years of experience.

Means and standard deviations were calculated on continuous data, including pre-test and posttest scores. A paired sample t-test was used to analyze if significant mean differences existed between the pre-test and post- test data. The results of this test were used to determine if there was a statistically significant difference between the pre-test and post-test. If participants had a significant increase from pre-test to post-test, the analysis would support the hypotheses that accuracy of medication identification would increase following simulation instruction.

\section{Results}

The data collected on the pre-test and post-test were used to determine if accuracy of medication error identification increased following the participation in one-hour simulation scenario.

\section{Demographics Analysis}

After completing a demographic analysis it was determined that all ten participants were LPNs with less than five years experience, who administer medication as part of their daily duties. Ninety percent $(\mathrm{N}=9)$ of the participants were female and ten percent $(\mathrm{N}=1)$ were male as presented in table 1 . The participants had between one and four years experience, with the median years of experience being 2.4 years.

\section{Data Analysis}

Pre-test percentage scores ranged between $78 \%$ and $90 \%$. The analysis revealed a mean percentage score of 84 with a standard deviation of $4.69(\mathrm{SD}=4.69)$ as displayed in table 2. This indicates that on average participants had a score or percentage of 84 . 


\begin{tabular}{|l|l|l|}
\hline Variable & Frequency & $\%$ \\
\hline Gender respondent & & \\
\hline Female & 9 & $90.0 \%$ \\
\hline Male & 1 & $10.0 \%$ \\
\hline Years of Experience & & \\
\hline 1 year & 2 & $20.0 \%$ \\
\hline 2 Years & 3 & $30.0 \%$ \\
\hline 3 Years & 4 & $40.0 \%$ \\
\hline 4 Years & 1 & $10.0 \%$ \\
\hline \multicolumn{2}{|l|}{ Table. 1 Demographic Variables Used in the Statistical Analyses } \\
\hline Variable Table. 2 Means and Standard Deviations, Focal Variables \\
\hline Pretest score & Mean & Std. Dev. \\
\hline Post-test score & 84.00 & 4.69 \\
\hline
\end{tabular}

Post-test results following one hour of simulation instruction revealed a percentage score range between $88 \%$ and $97 \%$. The analysis revealed a mean percentage score of 92.4 , with a standard deviation of 2.67. This indicates that the average post-test percentage score for the sample is 92.4 .

\section{Paired Samples t-Test}

A paired-samples t-test was conducted to compare pre-test scores before the simulation instruction and post-test scores following the simulation instruction. This is the appropriate method because a pair- sample t-test test for differences between the pretest and posttest score. The results are presented in Table 3 . There was a statistically significant difference in the pretest and posttest percentage scores $(\mathrm{t}=-8.53, \mathrm{p}=0.000)$. The posttest score was higher $(\mathrm{M}=92.40)$ than the pretest score $(\mathrm{M}=84.00)$. This result suggests that simulation instruction does have an effect on medication error identification. Specifically, the results suggest that medication error identification accuracy will increase when individuals participate in simulation instruction.

\begin{tabular}{|c|c|c|c|c|c|c|}
\hline \multirow[b]{2}{*}{ Variable } & \multicolumn{2}{|c|}{ Pretest } & \multicolumn{2}{|c|}{ Post-test } & \multirow[b]{2}{*}{$\mathrm{t}$} & \multirow[b]{2}{*}{$\mathrm{p}$} \\
\hline & M & SD & M & SD & & \\
\hline Scores & 84.00 & 4.69 & 92.40 & 2.67 & 8.53 & 0.000 \\
\hline
\end{tabular}

\section{Discussion}

The overall goal of this scholarly study was to evaluate the effect of simulation on medication identification accuracy in licensed practical nurses with less than five years experience. The project had three main objectives: (1) increase accuracy of medication identification and medication administration competency in licensed practical nurses (2) improve communication regarding recognized medication errors (3) decrease the occurrence and number of medication errors/near misses. The effect of simulation on medication error identification was measured using the SAM scale before and after the simulation activity.

The primary finding of this study showed that there was a statistically significant change in the participant scores on the posttest when compared to the pretest. There was a mean increase of 8.4 percent in scores among all participants. This indicated that the simulation may have an impact on medication error identification.

\section{Strengths}

This scholarly project had several strengths. One key one is that the study was the first reported study to focus on the effect of simulation on medication error identification in licensed practical nurses. The participant response rate was $100 \%$, as all participants who took the pretest also took the posttest. The tested reliability testing of the SAM scale was evaluated to be at an adequate level. The study results also suggest that simulation could be a plausible educational tool for novice licensed practical nurses.

\section{Limitations}

There are some limitations to the scholarly project. One limitation recognized in this scholarly project was the small sample size. A total of 10 participates were included in this study. This project was initiated in a very rural area, with a relatively small population of licensed practical nurses. It is likely that this reality has contributed to the small sample size. All participants were recruited from the same long-term care facility. This could have led to over generalization of results. Each participant completed the scenario on different days. Therefore, it is possible that participants may have shared information with subsequent participants.

Time, has proven to be another limitation of this scholarly project. Due to unforeseen delays in approval by the appropriate institutional review board (IRB), there has been less time allotted for recruitment. In addition, a longer testing period would allow for a follow up simulation instructional activity. Future plans for this project would include a follow up simulation with an added pre-briefing session.

\section{Implication for Future Nursing Practice}

Reliable means of reinforcing and assessing competency is important. Simulation instruction could be used for ongoing training for practicing nurses. These sessions will allocate participants an opportunity to discuss medication errors and the reasons they occur in attempt to discover the root-causes of these errors. In addition, the use of simulation could prove to be a plausible resource for the orientation of new nurses with little experience. According to the American 
Nurses Association (ANA ) [10] about 155,000 new graduates entered the workforce in 2015 (2016). Appropriate training and practice are essential to confidence, safe practice, and better patient outcomes. Simulation creates a safe environment for novice nurses to groom skills and learn from mistakes.

\section{Conclusion}

In conclusion, the results of this scholarly project will be used to provide evidence to support the use of simulation instruction to improve accuracy of medication administration and error identification of licensed practical nurses. It is crucial that the competency of novice nurses be evaluated and established in an effort to promote safe and quality patient care. As discussed simulation instruction could be implemented to present evidence-based knowledge on a ongoing basis which will provide these nurses with the most update to date information to improve accuracy and improve patient outcomes. By decreasing the mental, physical and financial cost experienced by patient as a result of medication errors, the nurse will in turn decrease the costs on the health care systems.

\section{References}

1. Institute of Medicine. (2010) The Safe Use Initiative and Health Literacy: Workshop Summary. Washington, DC: The National Academies Press

2. Elden NMK, Ismail A (2016) The Importance of Medication Errors Reporting in Improving the Quality of Clinical Care Services. Global J Health Sci 8: 243-251.

3. Barnsteiner J (2011) Teaching the culture of safety. Online J Issues in Nurs 16.

4. Charney W (2012) Epidemic of medical errors and hospitalacquired infections: systemic and social causes. Boca Raton: CRC Press.

5. Pronovost PJ, Cleeman JI, Wright D, Srinivasan A (2015) Fifteen years afterTo Err is Human: a success story to learn from: Table 1. BMJ Quality \& Safety 25: 396-399.

6. Weinger MB, Gaba DM (2014) Human Factors Engineering in Patient Safety. Anesthesiology 120: 801-806.

7. Collins SJ, Newhouse R, Porter J, Talsma A (2014) Effectiveness of the surgical safety checklist in correcting errors: a literature review applying Reason's Swiss cheese model. AORN J 100 :65-79.

8. Ryan DD (2007) Measurement of student nurse performance in the safe administration of medication (Order No. 3279892). Available from ProQuest Central; ProQuest Dissertations \& Theses Global. (304744036).

9. Gonzales Kelly J (2012) "Assessments of Safe Medication Administration in Nursing Education." J Nurs Edu Practice 2.1.

10. ANA Position Statement (2010): Position statement on just culture model. 